\begin{tabular}{c} 
Volume and Issues Obtainable at Center for Sustainability Research and Consultancy \\
Journal of Business and Social Review in Emerging Economies \\
ISSN: 2519-089X (E): 2519-0326 \\
Volume 5: No. 1, June 2019 \\
CSRᄃ \\
Journal homepage: www.publishing.globalcsrc.org/jbsee \\
\hline
\end{tabular}

\title{
A systematic synthesis of evidence regarding relationship of physical activity and sports participation with trait self-control
}

\author{
${ }^{1}$ Asif Ali, ${ }^{2}$ Muhammad Azam, ${ }^{3}$ Jawairyiah Mattiullah, ${ }^{4}$ Adeel Akhtar \\ ${ }^{1}$ Assistant Professor, Department of Physical Education \& Sports Science, The Islamia University of Bahawalpur, \\ Pakistan.goraya128@hotmail.com \\ ${ }^{2}$ Assistant Professor, Department of Physical Education, University of Gujrat, Pakistan. \\ ${ }^{3}$ Scholar, Virtual University, Islamabad, Pakistan. \\ ${ }^{4}$ Assistant Professor, Department of Commerce, Bahauddin Zakariya University, Multan, Pakistan. \\ adeel.akhtar@bzu.edu.pk
}

\begin{tabular}{l}
\multicolumn{1}{c}{ ARTICLE DETAILS } \\
\hline History \\
Revised format: May 2019 \\
Available Online: June 2019 \\
\\
Keywords \\
Self-Control, Exercise, Activity, \\
Prevention, Trait
\end{tabular}

JEL Classification:

J20, J29,R10, R11

\begin{abstract}
Individuals with low level of trait self-control exhibit several negative behaviors such as violence, addictive behaviors (drug addiction, technological addictions), suicide, unhealthy eating, and criminal behaviors. In contrast, high level of self-control thought to be associated with positive psychosocial and physical health related outcomes. Interventions contribute to boost self-control seems essential for individual with risk of low trait self-control.

The purpose of this study is to systematically evaluate the relationship between physical activity and trait self-control.

For this purpose, full-text articles were searched in research databases including Psych INFO, PubMed, Medline, The Cochrane Library, Wiley Library, Taylor \& Francis, Science Direct, Springer, ERIC, JSTOR, Google scholar, and Sage Journals. Following screening for the study selection criteria and assessment of risk of bias, twenty one studies (13cross-sectional, 5 longitudinal and 3 interventions) with low or moderate risks of bias were finally selected for qualitative synthesis. Findings from all of the included studies consistently showed that there was a significantly positive relationship between physical activity and trait self-control. It is suggested that increasing self-efficacy, decreasing aggression, and improvement in positive and negative emotional states might be underlying mechanisms working for beneficial effects of physical activity on trait self-control.

These suggest that physical activity and exercise may serve as a potential means to enhance trait self-control. Practical implementation and recommendations for policy makers are put forward in this connection.
\end{abstract}

(C) 2019 The authors, under a Creative Commons AttributionNonCommercial 4.0

Corresponding author's email address: adeel.akhtar@bzu.edu.pk

Recommended citation: Ali, A., Azam, M., Mattiullah, J. and Akhtar, A. (2019). A systematic synthesis of evidence regarding relationship of physical activity and sports participation with trait self-control. Journal of Business and Social Review in Emerging Economies, 5 (1),19-40

DOI: $10.26710 /$ jbsee.v5i1.627 


\section{Introduction}

The construct of trait self-control is considered significantly critical while determining influential factors effecting human behaviors.In the existing literature, considerable discrepancies exist concerning its conceptualization, assessment tools and procedures, and even its name. However,in general, current theories agreed upon the view that trait self-control can be conceptualized as the capacity of an individual to regulate successfully emotions, feelings, immediate tendencies, impulses, and behaviors, or to control predominant dispositions in an attempt to attain longterm goals in the later life(D. T. De Ridder \& Lensvelt-Mulders, 2018). In addition, trait self-control implies as the potential to exhibit consciously in wide range circumstance and situations that can be evaluated using self-reported questionnaires (Allom, Panetta, Mullan, \& Hagger, 2016; Tangney, Baumeister, \& Boone, 2004).

Research suggested that peoples varying with respect to their ability and level of trait self-control that resulting in exerting different impact oncertain behaviors, life style, attitudes and over all life of the individuals. For example, individuals with high trait self-control exhibit less criminal behavior (Bobbio, Arbach, \& Vazsonyi, 2019), less alcohol and fast food consumption, and healthy eating (Martin S. Hagger et al.). Moreover, research also, demonstrated positive association of trait self-control with stable emotions, self-esteem, being open, perspective taking and negatively related with shyness, personal distress, and unconcern (Pilarska \& Baumeister, 2018). Other studies indicated that individuals having higher level of self-control exhibited higher academic performance, good social relations, and higher psychological health(Tangney et al., 2004). In contrast,negative effects such as borderline personality symptoms (Hallquist, Hipwell, \& Stepp, 2015), depression and loneliness (Özdemir, Kuzucu, \& Ak, 2014), aggression and addictive behaviors (Kim, Namkoong, Ku, \& Kim, 2008), gambling disorder (von Hammerstein et al., 2018), burnout (Seibert, May, Fitzgerald, \& Fincham, 2016), loose temperament (Nęcka, Korona-Golec, Hlawacz, Nowak, \& Gruszka-Gosiewska, 2018), obesity and eating disorders (Mackenbach et al., 2019), and suicidal behavior (Baer et al., 2018) appeared to be related with low level of self-control potentials. In the same line, Malouf et al. (2014) found that there was inverse association of trait self-control with maladaptive behaviors including suicidal behavior, unhealthy sex behavior, and substance dependency in a sample of jail inmates.

These findings suggest that the trait self-control is a multi-facets and complex construct that is related with wide range of behaviors, thoughts, and emotions. Therefore, professionals in psychology, therapeutic experts dealing with psychiatric diseases, sociologist, educationist, and criminologists are deeply concerned with the issues generated due to lack of trait self-control (Piquero, Jennings, Farrington, Diamond, \& Gonzalez, 2016). In line with above discussions, it seems crucially important to identify the therapeutic interventions, and ways that could help improve self-control in wide range of populations. The experts have been showing great concern regarding therapeutic interventions to deal with issue of low self-control on their target populations. The interventions, approaches, and strategies focusing on developing the skills that regulate emotions, behaviors, cognition and promote problem solving and social skills have importance for considerations in this regard (Crick \& Dodge, 1994; Elias, Zins, \& Weissberg, 1997; Stein, Thurston, \& Mather, 1997). Interventions such as mindfulness training (Rowland, Wenzel, \& Kubiak, 2016), situational self-control strategies (Duckworth, Gendler, \& Gross, 2016), cognitive-behavioral therapy (Scarpa \& Reyes, 2011), relaxation training, cognitive coping strategies, video tape training/role playing interventions, and immediate/delayed rewards clinical interventions(Piquero et al., 2016), have been the main focus of previous research in an efforts to improve trait self-control. On the other hand, researchers are curious to explore more alternative approaches such as physical activity and exercise(Scully, Kremer, Meade, Graham, \& Dudgeon, 1998).

Certain level of physical activity and exercise is considered important to constitute several psychological, social, cognitive, and health related benefits that may contribute dealing with self-control concerns. In this connection, previous research showed that an optimal level of physical activity appeared to reduce obesity and poor eating (Fan \& Jin, 2013), depression, anxiety, psychotic symptoms, and suicidal behavior(Tao et al., 2007). Moreover, sufficient amount of physical activity is suggested to promote good eating habits, fulfilling the commitments, emotional control, timely completing the household chores, financial spending control, improvement in study habit and reduced negative behaviors such as perceived stress, alcohol, smoking, emotional distress, and caffeine consumption (Oaten \& Cheng, 2006).Exercise has also been suggested to foster self-confidence, self-esteem,selfworth, controlling deviant tendencies, problem solving ability, happiness, self-discipline, pleasant mood, and socialization(Lubans, Plotnikoff, \& Lubans, 2012; Nieman, 2002).Previous research has also corroborated that athletes encounter various challenges that require utilization of self-control capacity.It is also known that positive psychological states (happiness, pleasant mood) can result in promoting self-control (D. De Ridder \& Gillebaart, 
2017; Isen \& Reeve, 2005). In addition, involvement in physical activity improves characteristics and skills those are essential for self-control abilities. On the other hand, the negative psychological characteristics are related with low self-control can be improved through physical activity. Therefore, this discussion theoretically led to assume that physical activity may be an effective source to improve trait self-control.

Furthermore, lack of involvement in physical activity has become global health concern in recent decades. A large proportion of the people do not meet the basic requirement of participation level in physical activity in almost all of the countries of the world. This lead to prevalence of psychological disorders, physical diseases, social problems, and exerting extra load on nation's economies(Penedo \& Dahn, 2005). In the same vein, if, in some way, selfcontrol has some relationship with health related behaviors including physical activity or unhealthy behaviors such as sedentary behavior, as it has consistently been demonstrated in the past research (D. T. De Ridder \& LensveltMulders, 2018). Then, plausibly it points to the assessment of the efficacy of this relationship. This would be useful for policy makers to make decisions regarding promotion of positive health and nullifying negative health behaviors in the communities. Therefore, the purpose of this review is to assess the relationship of physical activity, exercise, and sports participation with trait self-control. To date, as per our knowledge, no review has systematically presented findings from published work on this topic. This review essentially would add quality based knowledge to the policy makers, psychologist, educationists, and health related professional for making decisions based on evidence based recommendations.

\section{Methodology}

\subsection{Study Inclusion and Exclusion Criteria}

Inclusion criteria was as follows: 1) the studies with the objective of assessing relationship of physical activity, exercise, sports participation, sedentary behavior, physical fitness on self-control either as a primary focus or as a sub theme, 2) cross-sectional, cohort, longitudinal, experimental, case-control, randomized control trials, and intervention research studies, 3)studies involved all sample types including students, community and healthy or patient samples, 4) studies used participants with any age (e.g., children, adolescents , adults, and old)that have both male and female genders, 5) studies in English language and conducted in any country/nation, 6)studies included physical activity-related keywords (e.g., physical activity, exercise, sports participation, physical fitness, sedentary behavior, athletes or non-athletes) and the keyword of self-control either in titles or in abstracts, 7) studies measured trait-self-control, and 8) studies published in peer review journals. Studies were excluded if they failed to meet the following criteria: 1) full texts were not available, 2) studies did not measure physical activity, exercise, sports participation, sedentary behavior, physical fitness or self-control3) studies presented findings solely based on behavioral measures of self-control. However, study is included if it used combination of both self-reported and behavioral measures of self-control. There is less agreement among the researchers on the use of behavioral measures of self-control(Allom et al., 2016), 4) studies presented insufficient data regarding the objectives of this review, 5) unpublished studies, commentaries, letters to the editor, conference proceedings, books and book chapters, thesis and dissertations, 6) case-reports, published reviews, case-series, qualitative studies, pilot studies, 7) studies lacking sufficient methodological details, and 8)studies used self-regulation as synonyms to self-control. Since, the constructs of self-control and self-regulation have not been considering the same in research literature, therefore, we excluded the studies used self-regulation approach as synonym to the concept of self-control (Gillebaart, 2018; Mann, De Ridder, \& Fujita, 2013).

\subsection{Search Strategy}

Systematic search was conducted (December 2018) in major research data bases including Psych INFO, PubMed, Medline, The Cochrane Library, Wiley Library, Taylor \& Francis, Science Direct, Springer, ERIC, JSTOR, Google scholar, and Sage Journals. Search terms consisted of "physical activity" AND "self-control", "exercise" AND "self-control", "physical fitness" AND "self-control", "sedentary behavior" AND "self-control", "athletes versus non-athletes" AND "self-control", and "sports participation" AND "self-control". In addition, reference lists of the research articles on the topics related to sport, exercise, and physical activity on self-control were screened to search for additional articles.

\subsection{Study Selection}

Based on inclusion and exclusion criteria, two experts independently performed extensive review to select the studies for this review. The experts performed review in following two steps: firstly, they screened for titles and abstracts of the retrieved studies looking for information and data relevant to the objectives of this review. And secondly, full texts of the retrieved articles were also reviewed for potential relevancy. Three meetings were 
conducted among experts to discuss and resolve any discrepancy regarding study selection. Differences of opinion regarding selection of studies were resolved through discussions between both experts. A third expert was consulted in case the disagreement still existed between the two experts. Figure 1 illustrates the full details of study selection procedure.

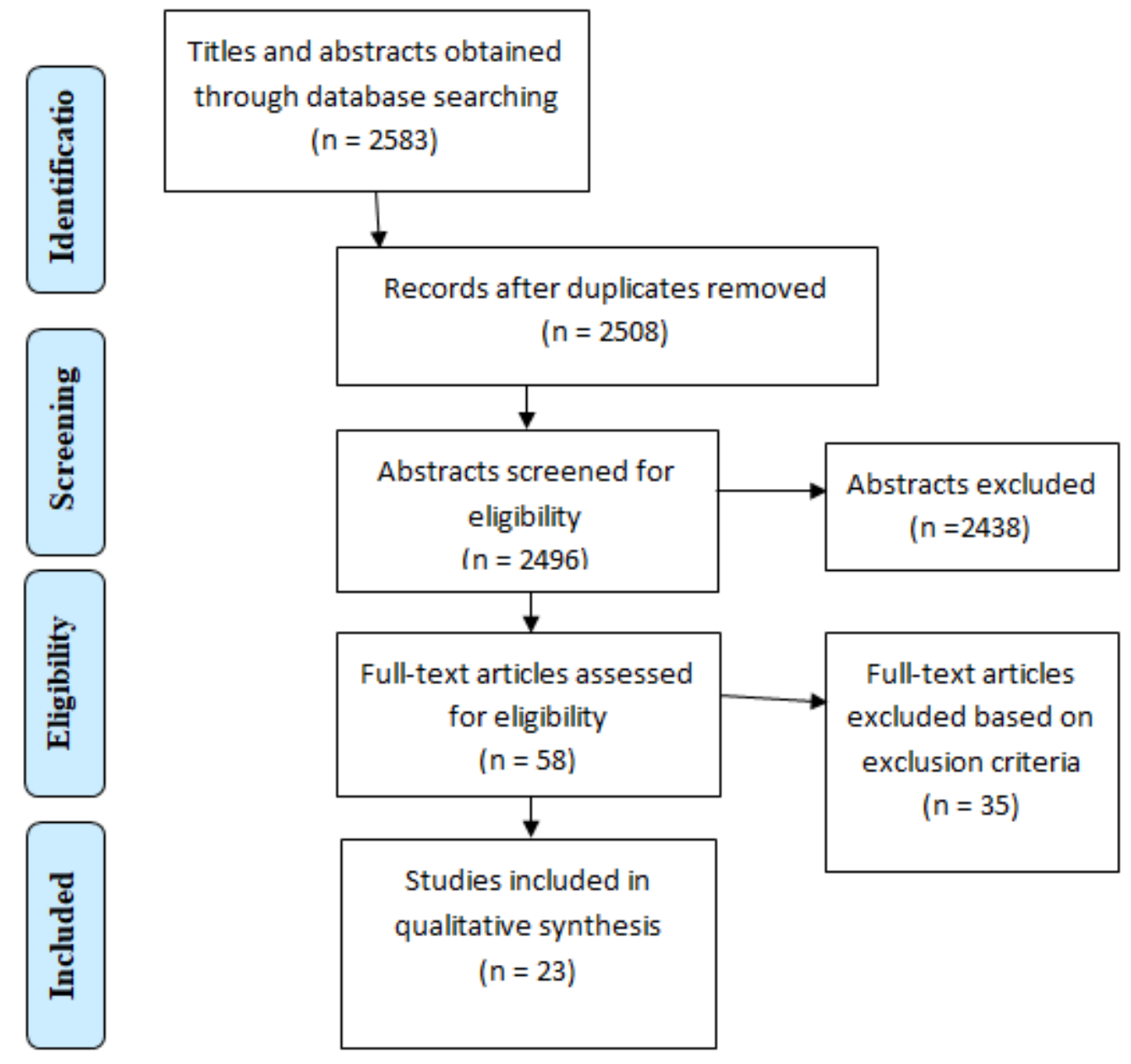

Figure 1. Search strategy and selection of studies

\subsection{Assessment of Risk Of Bias}

Two independent reviewers assessed the risk of bias for the studies met inclusion and exclusion criteria. Quality of the cross-sectional studies was evaluated using The Newcastle-Ottawa Scale (NOS) adapted for cross-sectional studies (Herzog et al., 2013).The Effective Public Health Practice Project (EPHPP) Quality Assessment Tool for Quantitative Studies was used for appraisal of risk of bias of longitudinal and intervention studies (Project, 1998). This tool is considered valid and reliable for quality appraisal of cohort, longitudinal, randomized control trials, time series, control clinical design, and case-control studies and have been widely used in previous research(Armijo-Olivo, Stiles, Hagen, Biondo, \& Cummings, 2012; Thomas, Ciliska, Dobbins, \& Micucci, 2004). Quality ratings of the eligible studies were performed based on the scoring system prescribed by EPHPP reviewer's dictionary and manual. Based on the appraisal, the selected studies were classified as weak (two or more weak ratings), moderate (one weak rating), and strong (no weak ratings) categories. Discrepancies regarding the quality ratings for admissible publications was resolved through discussions between the two experts and agreement level was determined with a Kappa $=.82$.

\section{Results}

\subsection{Study Selection}

2496 titles and abstracts were carefully screened for admissibility. After removing ineligible abstracts and titles, 58 full text articles were retrieved and considered for further screening. 35 studies were excluded due to not meeting 
inclusion criteria for this review and finally 26 articles were selected for quality assessment in full text.Of these, 13 cross-sectional studies were categorized as very good, good or satisfactory. 5 longitudinal studies and 3 intervention studies having low or moderate risk of bias were selected for qualitative synthesis.

\subsection{Study Characteristics}

\subsubsection{Cross-Sectional Studies $(\mathrm{N}=13)$}

We found thirteen eligible cross-sectional studies that examined association or relationship of physical activity or physical fitness with trait self-control. The details about characteristics of the selected studies are presented in table 1.A. Study characteristics were extracted following careful reviewing of the selected studied by two experts. These characteristics included author name and year of publication, population, sample size, sample selection procedure, participant's ages and gender, physical activity and trait self-control measures, data analysis tests, and results and findings.

The total number of participants consisted of 7309 ranging from 134 to 2812 participants in these studies. Five studies were performed in adolescents from school, colleges, or university students and eight studies in adults. Five studies included both adolescents and adults participants. Participant's ages ranged from 12 to 65 years in these studies. Among thirteen admissible cross-sectional studies, two studies included only male participants whereas remaining eleven studies included both male and female participants.Majority of the studies used convenience sampling while a few studies used purposive (Tedesqui \& Young, 2017) or cluster sampling (Chen, Li, Xie, Li, \& Zhang, 2018; Chen et al., 2019)procedures. None of the studies used random or systematic sampling procedure for sample selection. The response rates of the participants were greater than $80 \%$ in Ten out of thirteen studies. Less than half of the studies (5/13) controlled for almost all of the important confounding factors, whereas, approximately half of the eligible studies (6/13) controlled for the most important confounding factors (e.g., age, sex), except two studies that did not report controlling of confounding variables.

In particular, three studies included professional athletes, one study selected participants from fitness gym, and one recruited participants from soldiers attending physical training. All of these studies used self-reported valid and reliable methods/ tools of measuring sports participation, physical activity, exercise, and trait self-control. However, only one study administered objective measures of physical activity. Majority of these studies(8/13) used either short or long version of self-control scale developed by Tangney et al. (2004). In these studies, three were conducted in Netherlands, two in USA, two in China, and one in each of the countries including Finland, Israel, Norway, Canada, South Korea, and UK. All of the thirteen cross-sectional studies were found to have significantly positive relationship or association of sports participation, physical activity, exercise, or physical fitness with traitself-control.

\subsubsection{Longitudinal $(\mathrm{N}=5)$ and Intervention Studies $(\mathrm{N}=3)$}

We found five longitudinal studies assessed association between physical activity and trait self-control. Table 1.B and $\mathrm{C}$ illustrates characteristics of the longitudinal and intervention studies included in this article. These studies consisted of 30 to 3993 participants and included 7153 participants in total. Three studies included participants from university student's population and two studies were conducted in general population. Participant's ages were ranged from 15 to 45 years, respectively. The follow up period varied from 2 weeks to 6 years across the five longitudinal studies. Only one study used random sampling approach while reaming four studies used convenience sampling procedures, however, they did justify the sample size. Four out of five studies used valid and reliable physical activity measures. All of the studies used valid and reliable trait self-control measures. These studies also did control for the most important confounding factors either in designs or in analysis. These studies were conducted in Germany $(\mathrm{N}=2)$, Netherlands $(\mathrm{N}=1)$, Finland $(\mathrm{N}=1)$, and Canada $(\mathrm{N}=1)$. All of these studies reported significantly positive relationship between physical activity and trait self-control.

We identified three studies with intervention research paradigms assessing association between physical activity and trait self-control. The major characteristics of the three intervention studies are described in Table 1.C.Of these, one study applied randomized control trials(Cecchini, Montero, Alonso, Izquierdo, \& Contreras, 2007), one study controlled clinical trial(Shachar, Ronen-Rosenbaum, Rosenbaum, Orkibi, \& Hamama, 2016) and one study had time series design(Will Crescioni et al., 2011). Participant's ages were ranged from 9 to 60 years in these studies. Participants of two studies consisted of school children and participants of one study were selected from university students and general population. These studies included 921 participants in total that ranged from 86 to 649 participants. The intervention period ranged from 5 to 24 weeks, respectively. Of the three intervention studies, two 
used team sports as intervention strategy and one study used general physical exercise program (e.g., walking, jogging, aerobic dance, cycling, and swimming). All of the three studies measured trait self-control with valid and reliable tools. One study was conducted in USA, one in Israel, and one in Spain, respectively.All of the three studies had low risk of bias and had overall strong rating. Findings from the intervention studies consistently showed significantly positive relationship of sports participation, exercise, and trait self-control.

Table 1. A. characteristics of cross-sectional studies assessing relationship of physical activity and trait selfcontrol

\begin{tabular}{|c|c|c|c|c|c|c|c|}
\hline $\begin{array}{l}\text { Autho } \\
\text { r/ } \\
\text { countr } \\
\text { y }\end{array}$ & $\begin{array}{l}\text { Population, sample, } \\
\text { Participants ages }\end{array}$ & $\begin{array}{l}\text { Male } \\
\%\end{array}$ & $\begin{array}{l}\text { Res } \\
\text { pon } \\
\text { se } \\
\text { rate } \\
(\%)\end{array}$ & $\begin{array}{l}\mathrm{PA} \\
\text { measure }\end{array}$ & $\begin{array}{l}\text { Trait } \\
\text { self- } \\
\text { control } \\
\text { measure }\end{array}$ & Analysis & Results/ findings \\
\hline $\begin{array}{l}\text { (Wills } \\
\text {, et al,. } \\
2007 \text { ) } \\
\text { USA }\end{array}$ & $\begin{array}{l}\begin{array}{l}\text { sample of } \\
\text { selected from three }\end{array} \\
\text { public high schools } \\
\text { with mean } \\
\text { age } 14.6 \text { years } \text { (SD } \\
0.7)\end{array}$ & 52 & 83 & $\begin{array}{l}\text { Vigorous } \\
\text { exercise, } \\
\text { sports } \\
\text { participati } \\
\text { on and } \\
\text { sedentary } \\
\text { behavior } \\
\text { were } \\
\text { measured } \\
\text { through } 4 \\
\text { items } \\
\text { selected } \\
\text { from valid } \\
\text { and } \\
\text { reliable } \\
\text { measures }\end{array}$ & $\begin{array}{l}12 \text { items } \\
\text { from } \\
\text { multiple } \\
\text { measure } \\
\mathrm{s} \text { of self- } \\
\text { control } \\
\text { driven } \\
\text { from } \\
\text { past } \\
\text { research }\end{array}$ & $\begin{array}{l}\text { confirmatory } \\
\text { analysis, } \\
\text { correlations, } \\
\text { and } \\
\text { structural } \\
\text { equation } \\
\text { modeling } \\
\text { analysis }\end{array}$ & $\begin{array}{l}\text { Trait self-control were } \\
\text { positively related with } \\
\text { consumption of } \\
\text { healthy eating, spots } \\
\text { participation, and } \\
\text { negatively related with } \\
\text { sedentary behavior. } \\
\text { Poor trait self-control } \\
\text { exhibited decreased } \\
\text { vigorous exercise. }\end{array}$ \\
\hline
\end{tabular}




\begin{tabular}{|c|c|c|c|c|c|c|c|}
\hline $\begin{array}{l}\text { Junge } \\
\text { r,et } \\
\text { al., } \\
\text { 2010) } \\
\text { Nather } \\
\text { land }\end{array}$ & $\begin{array}{l}\text { sample consisted of } \\
201 \text { adolescents using } \\
\text { convenience sampling } \\
\text { with ages between } 15 \\
\text { and } 20 \text { years }\end{array}$ & 50 & 100 & $\begin{array}{l}2 \quad \text { times } \\
\text { selected } \\
\text { from valid } \\
\text { and } \\
\text { reliable } \\
\text { Moderate- } \\
\text { to- } \\
\text { Vigorous } \\
\text { Physical } \\
\text { Activity } \\
\text { measure }\end{array}$ & $\begin{array}{l}\text { Tangney } \\
\text { 's SCS }\end{array}$ & $\begin{array}{l}\text { Stepwise } \\
\text { regression } \\
\text { analysis, } \\
\text { Pearson } \\
\text { correlations, } \\
\text { hierarchal } \\
\text { regression } \\
\text { analysis } \\
\text { and a } \\
\text { multivariate } \\
\text { step-wise } \\
\text { analysis }\end{array}$ & $\begin{array}{l}\text { Trait self-control }(\beta= \\
.21) \text { was positively } \\
\text { related with physical } \\
\text { activity but negatively } \\
\text { related with BMI }(\beta= \\
-.17) \text {. }\end{array}$ \\
\hline $\begin{array}{l}\text { (Kinn } \\
\text { unen, } \\
\text { et al,. } \\
\text { 2012) } \\
\text { Finlan } \\
\text { d }\end{array}$ & $\begin{array}{l}\text { Sample sizes was } 482 \\
\text { men ages ranged from } \\
18-29 \text { years }(\mathrm{M}=20 \\
\text { yeras })\end{array}$ & 100 & 47 & $\begin{array}{l}\text { Height, } \\
\text { weight, } \\
\text { and waist } \\
\text { circumfere } \\
\text { nce. } \\
\text { Leisure } \\
\text { Time } \\
\text { Physical } \\
\text { Activity, } \\
\text { BMI, } \\
\text { Cooper } \\
\text { 12- } \\
\text { Minutes } \\
\text { Running } \\
\text { Test. } \\
\text { Muscle } \\
\text { fitness } \\
\text { tests } \\
\text { included } \\
\text { pull-ups, } \\
\text { sit-ups, } \\
\text { standing } \\
\text { long jump } \\
\text { push-ups, } \\
\text { and a } \\
\text { back- } \\
\text { muscle test }\end{array}$ & $\begin{array}{l}\text { Tangney } \\
\text { 's SCS }\end{array}$ & $\begin{array}{l}\text { Linear } \\
\text { regression } \\
\text { analyses, and } \\
\text { Sobel test. }\end{array}$ & $\begin{array}{l}\text { Trait self-control was } \\
\text { positively associated } \\
\text { with leisure time } \\
\text { physical activity, } \\
\text { muscle fitness, aerobic } \\
\text { fitness, and negatively } \\
\text { related with BMI. }\end{array}$ \\
\hline $\begin{array}{l}\text { (Shac } \\
\text { haf, et } \\
\text { al., } \\
\text { 2014) } \\
\text { Isreal }\end{array}$ & $\begin{array}{l}\text { Sample comprised of } \\
491 \text { 10th-11th- and } \\
12 \text { th grade Israeli } \\
\text { high schools students. } \\
\text { They were divided in } \\
\text { to three comparison } \\
\text { groups including } 170 \\
\text { in sports group and } \\
185 \text { in non-sports but }\end{array}$ & 44 & 100 & $\begin{array}{l}\text { Competiti } \\
\text { ve sports } \\
\text { group, } \\
\text { physical } \\
\text { fitness } \\
\text { group, no } \\
\text { activity } \\
\text { group, }\end{array}$ & $\begin{array}{l}\text { Rosenba } \\
\text { um,s } \\
\text { SCS }\end{array}$ & $\begin{array}{l}\text { One-way } \\
\text { ANOVA }\end{array}$ & $\begin{array}{l}\text { The participants in the } \\
\text { sport group reported } \\
\text { greater self-efficacy, } \\
\text { attribution style, and } \\
\text { self-control compared } \\
\text { with the subjects in } \\
\text { the no-activity and } \\
\text { physical fitness } \\
\text { conditions. }\end{array}$ \\
\hline
\end{tabular}




\begin{tabular}{|c|c|c|c|c|c|c|c|}
\hline & $\begin{array}{l}\text { fitness program } \\
\text { group, and } 106 \text { in no } \\
\text { activity group. }\end{array}$ & & & & & & \\
\hline $\begin{array}{l}\text { (Toeri } \\
\text { ng, et } \\
\text { al., } \\
2015) \\
\text { Norwa } \\
\text { y }\end{array}$ & $\begin{array}{l}639 \text { male players } \\
\text { from soccer teams } \\
\text { took part in this study. } \\
\text { Participants ages were } \\
\text { ranged from } 15 \text { to } 38 \\
\text { years }(\mathrm{M}=23.6, \mathrm{SD}= \\
4.8) .\end{array}$ & 100 & 96 & $\begin{array}{l}\text { Soccer } \\
\text { players } \\
\text { were } \\
\text { divided in } \\
\text { to groups } \\
\text { based on } \\
\text { their } \\
\text { performan } \\
\text { ce and } \\
\text { experience } \\
\text {. }\end{array}$ & $\begin{array}{l}\text { Tangney } \\
\text { 's SCS }\end{array}$ & $\begin{array}{l}\text { chi-square } \\
\text { statistic, } \\
\text { confirmatory } \\
\text { factor } \\
\text { analysis, } \\
\text { exploratory } \\
\text { factor } \\
\text { analysis, } \\
\text { Multiple } \\
\text { regression } \\
\text { analyses, } \\
\text { Pearson } \\
\text { correlation }\end{array}$ & $\begin{array}{l}\text { Players with higher } \\
\text { performance and } \\
\text { players spending more } \\
\text { time in practice } \\
\text { exhibited significantly } \\
\text { higher trait self- } \\
\text { control. }\end{array}$ \\
\hline $\begin{array}{l}\text { (Tedes } \\
\text { qui, et } \\
\text { al., } \\
\text { 2017) } \\
\text { Canad } \\
\text { a }\end{array}$ & $\begin{array}{l}\text { Online survey study } \\
\text { used }, \quad \text { purposive } \\
\text { sampling to select } \\
244 \text { athletes ( } \mathrm{M} \text { age } \\
=21.96 \text { years, range }= \\
12-43 \text { years), }\end{array}$ & 53 & 100 & $\begin{array}{l}\text { Athlete's } \\
\text { level of } \\
\text { skill and } \\
\text { playing } \\
\text { practice } \\
\text { were } \\
\text { assessed } \\
\text { through } \\
\text { valid and } \\
\text { reliable } 7 \\
\text { item valid } \\
\text { tool. }\end{array}$ & $\begin{array}{l}\text { Tangney } \\
\text { 's SCS }\end{array}$ & $\begin{array}{l}\text { confirmatory } \\
\text { factor } \\
\text { analyses, } \\
\text { Partial } \\
\text { correlations, } \\
\text { two separate } \\
\text { one-way } \\
\text { analyses of } \\
\text { covariance. }\end{array}$ & $\begin{array}{l}\text { Enhanced trait self- } \\
\text { control was related } \\
\text { with higher level of } \\
\text { sport participation } \\
\text { commitment and more } \\
\text { time spending in sport } \\
\text { practice. }\end{array}$ \\
\hline
\end{tabular}




\begin{tabular}{|c|c|c|c|c|c|c|c|}
\hline $\begin{array}{l}\text { (Chen, } \\
\text { et al., } \\
2018) \\
\text { China }\end{array}$ & $\begin{array}{l}210 \text { national level } \\
\text { Chinese boxers were } \\
\text { recruited through } \\
\text { cluster sampling. } \\
\text { Participants' average } \\
\text { age was } 18.89 \text { years } \\
\text { while their average } \\
\text { experience was } 4.93 \\
\text { years. }\end{array}$ & 36.2 & 91 & $\begin{array}{l}\text { Level and } \\
\text { experience } \\
\text { of boxers } \\
\text { were } \\
\text { measured } \\
\text { through } \\
\text { valid and } \\
\text { reliable } \\
\text { tool. }\end{array}$ & $\begin{array}{l}\text { Self- } \\
\text { control } \\
\text { question } \\
\text { naire for } \\
\text { Chinese } \\
\text { athletes }\end{array}$ & $\begin{array}{l}\text { regression } \\
\text { coefficients }\end{array}$ & $\begin{array}{l}\text { The level of trait self- } \\
\text { control was high in } \\
\text { national boxers of } \\
\text { china. Self-control was } \\
\text { significantly greater as } \\
\text { a function of athlete's } \\
\text { level of competitions. }\end{array}$ \\
\hline $\begin{array}{l}\text { (Chen, } \\
\text { et al., } \\
\text { 2019) } \\
\text { China }\end{array}$ & $\begin{array}{l}\text { The sample size was } \\
414 \text { Chinese } \\
\text { Professional boxers } \\
\text { those were selected } \\
\text { using cluster } \\
\text { sampling. average age } \\
\text { of the sample was } \\
17.72 \text { years. }\end{array}$ & 58 & 92 & $\begin{array}{l}\text { Level and } \\
\text { years of } \\
\text { experience } \\
\text { was } \\
\text { measured } \\
\text { through } \\
\text { self- } \\
\text { reported } \\
\text { questions }\end{array}$ & $\begin{array}{l}\text { Self- } \\
\text { control } \\
\text { Questio } \\
\text { nnaire } \\
\text { for } \\
\text { athletes }\end{array}$ & $\begin{array}{l}\text { correlation } \\
\text { analysis, } \\
\text { variance } \\
\text { analysis, } \\
\text { regression } \\
\text { analysis, } \\
\text { Structural } \\
\text { equation } \\
\text { modeling, }\end{array}$ & 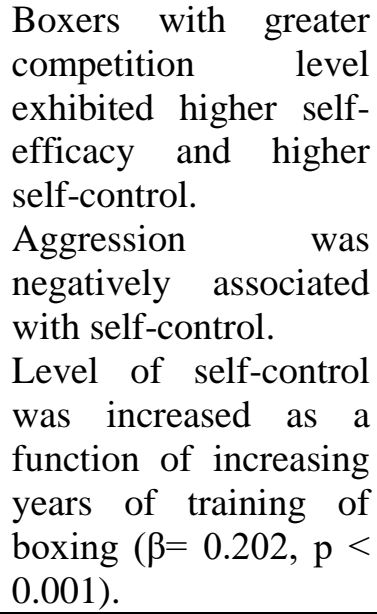 \\
\hline $\begin{array}{l}\text { (Briki, } \\
\text { w., } \\
2016) \\
\text { USA }\end{array}$ & $\begin{array}{l}\text { Sample consisted of } \\
317 \text { American adults } \\
\text { (Mean age } 32.97 \\
\text { years , SD age } \\
\text { D11.30), }\end{array}$ & 29.7 & 100 & $\begin{array}{l}\text { Self- } \\
\text { reported } \\
\text { regular } \\
\text { exercise, } \\
\text { BREQ-2 }\end{array}$ & $\begin{array}{l}\text { Tangney } \\
\text { 's SCS }\end{array}$ & $\begin{array}{l}\text { Correlation } \\
\text { Analyses, } \\
\text { Structural } \\
\text { model } \\
\text { analysis }\end{array}$ & 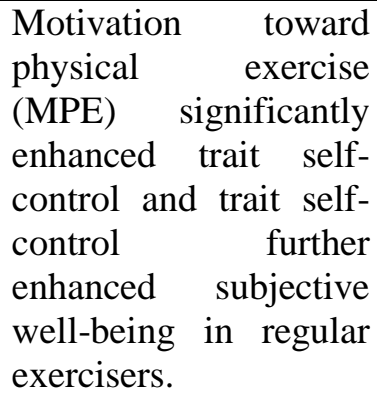 \\
\hline
\end{tabular}




\begin{tabular}{|c|c|c|c|c|c|c|c|}
\hline $\begin{array}{l}\text { (Gille } \\
\text { baart, } \\
\text { at al., } \\
2017) \\
\text { Nether } \\
\text { land }\end{array}$ & $\begin{array}{l}\text { online survey, } \\
\text { The sample was } 134 \\
\text { self-reported } \\
\text { exercisers that were } \\
\text { selected from fitness } \\
\text { gyms. Participants } \\
\text { ages ranged from 18- } \\
65 \text { years, Mean age } \\
\text { of } 37.93 \text { years (SD = } \\
12.44 \text {. }\end{array}$ & 38.8 & 100 & $\begin{array}{l}\text { Regular } \\
\text { exercisers } \\
\text { in gym. } \\
\text { SRHI }\end{array}$ & $\begin{array}{l}\text { Tangney } \\
\text { 's SCS }\end{array}$ & $\begin{array}{l}\text { Spearman's } \\
\text { rank-order } \\
\text { correlations, } \\
\text { Pearson } \\
\text { product- } \\
\text { moment } \\
\text { Correlations, } \\
\text { regression } \\
\text { analysis }\end{array}$ & $\begin{array}{l}\text { Trait self-control was } \\
\text { associated with } \\
\text { increased duration of } \\
\text { exercise }(r=0.33, p< \\
0.001) \text { and strength of } \\
\text { exercise habit ( } r \\
=0.31, p<0.001) \text {. }\end{array}$ \\
\hline $\begin{array}{l}\text { (Park, } \\
\text { et al., } \\
2016) \\
\text { South } \\
\text { Korea }\end{array}$ & $\begin{array}{l}\text { Convenience } \\
\text { sampling, } \\
\text { students were selected } \\
\text { through convenience } \\
\text { sampling from two } \\
\text { middle and two high } \\
\text { schools of south } \\
\text { Korea. Participant's } \\
\text { ages were ranged } \\
\text { from 15-18 years. }\end{array}$ & 17.7 & 89 & $\begin{array}{l}\text { Sports } \\
\text { participati } \\
\text { on } \\
\text { questionna } \\
\text { ire }\end{array}$ & SCRS & $\begin{array}{l}\text { Confirmator } \\
\text { y Factor } \\
\text { Analysis, } \\
\text { Structural } \\
\text { equation } \\
\text { modeling, } \\
\text { independent } \\
\text { t-test and } \\
\text { one way } \\
\text { analysis of } \\
\text { variance }\end{array}$ & $\begin{array}{l}\text { Sports participation } \\
\text { significantly and } \\
\text { positively influenced } \\
\text { self-control }(\beta=.154, p \\
<.05) \text {. }\end{array}$ \\
\hline $\begin{array}{l}\text { (Been } \\
\text { ackers } \\
\text {, et al., } \\
2017) \\
\text { Nether } \\
\text { land }\end{array}$ & $\begin{array}{l}2812 \text { adults with ages } \\
\text { ranged from } 25-75 \\
\text { years were surveyed. } \\
\text { Age mean and SD } \\
\text { 48.8-14.9. }\end{array}$ & 44.8 & $\begin{array}{l}45 . \\
5\end{array}$ & SQUASH & $\begin{array}{l}\text { Tangney } \\
\text { 's SCS }\end{array}$ & $\begin{array}{l}\text { linear } \\
\text { regression } \\
\text { with a log- } \\
\text { link } \\
\text { function and } \\
\text { generalized } \\
\text { linear } \\
\text { regression } \\
\text { models, }\end{array}$ & $\begin{array}{l}\text { High trait self-control } \\
\text { was related with } \\
\text { increased physical } \\
\text { activity. }\end{array}$ \\
\hline
\end{tabular}




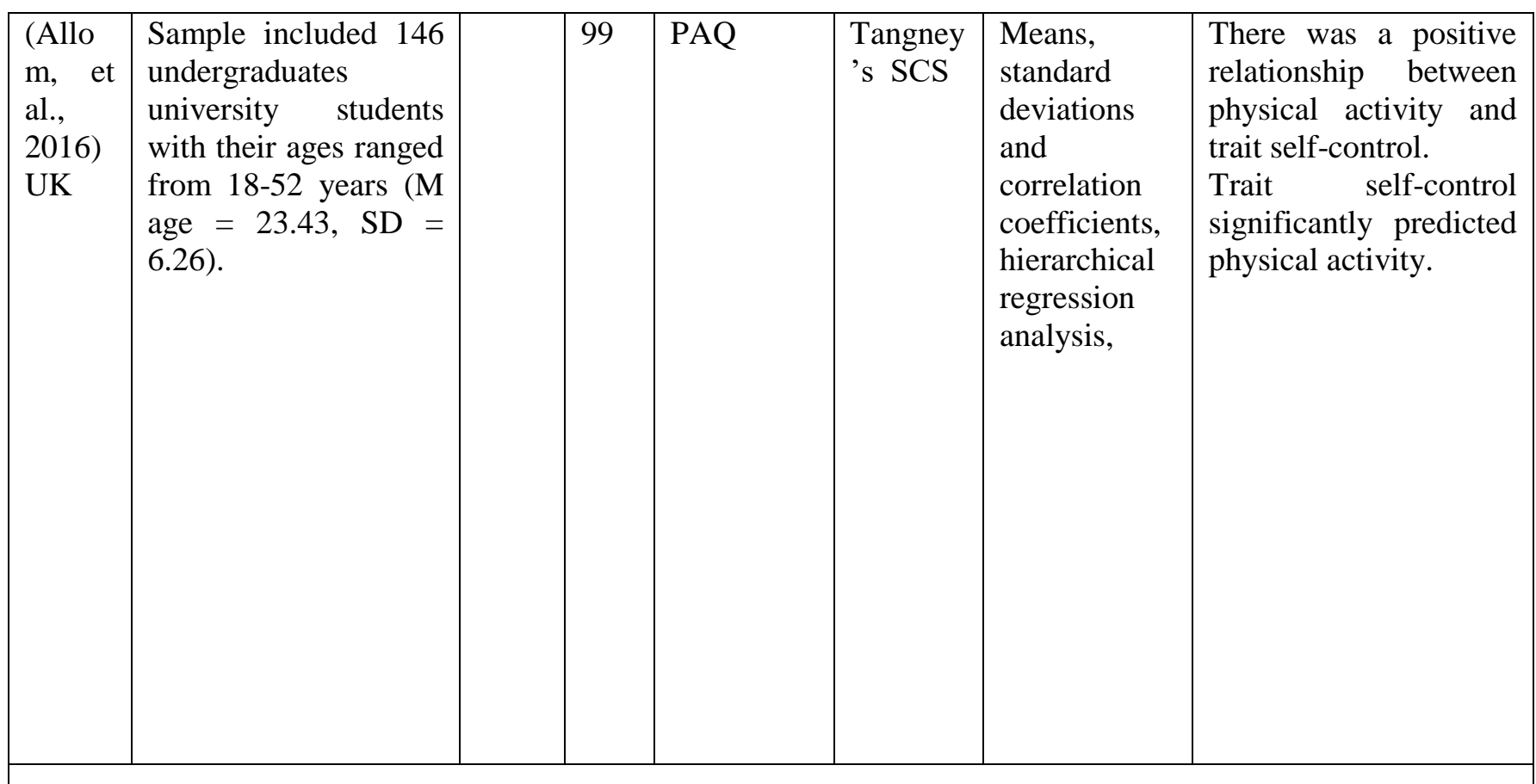

Table 1. B. Characteristics of longitudinal studies evaluated relationship between physical activity and trait selfcontrol.

\begin{tabular}{|c|c|c|c|c|c|c|c|}
\hline $\begin{array}{l}\text { Autho } \\
\text { r/ } \\
\text { countr } \\
\mathrm{y}\end{array}$ & $\begin{array}{l}\text { Population, sample size and } \\
\text { ages, follow up period }\end{array}$ & $\begin{array}{l}\text { Mal } \\
\mathrm{e} \%\end{array}$ & $\begin{array}{l}\text { Ret } \\
\text { urn } \\
\text { rate } \\
\%\end{array}$ & PA measures & $\begin{array}{l}\mathrm{SC} \\
\text { measures }\end{array}$ & Analysis & Findings \\
\hline $\begin{array}{l}\text { (Scho } \\
\text { ndube, } \\
\text { et al., } \\
\text { 2017) } \\
\text { Germa } \\
\text { ny }\end{array}$ & $\begin{array}{l}\text { One group pre-post } \\
\text { longitudinal design using } \\
\text { convenience sampling in } \\
\text { which } \\
63 \text { university students ages } \\
\text { ranged from } 19 \text { to } 32 \text { years } \\
(\mathrm{M}=23.5 ; \mathrm{SD}=2.8) \text { were } \\
\text { participated. Selected } \\
\text { variables were measured } \\
\text { consecutively for } 20 \text { days. }\end{array}$ & $\begin{array}{l}33 . \\
3\end{array}$ & $\begin{array}{l}83 . \\
3\end{array}$ & $\begin{array}{l}\text { Self-reported } \\
\text { physical } \\
\text { exercise } \\
\text { participation } \\
\text { for } 20 \text { days } \\
\text { using single } \\
\text { item valid } \\
\text { tool. }\end{array}$ & $\begin{array}{l}\text { Tangney's } \\
\text { SCS }\end{array}$ & $\begin{array}{l}\text { estimated } \\
\text { multileve } \\
1 \\
\text { models } \\
\text { analysis }\end{array}$ & $\begin{array}{l}\text { Reduction in } \\
\text { physical activity } \\
\text { was significantly } \\
\text { lower in the } \\
\text { participants } \\
\text { showed higher } \\
\text { trait self-control } \\
\text { when compared } \\
\text { with the } \\
\text { participants } \\
\text { exhibited lower } \\
\text { trait self-control. }\end{array}$ \\
\hline
\end{tabular}




\begin{tabular}{|c|c|c|c|c|c|c|c|}
\hline $\begin{array}{l}\text { (Droo } \\
\text { mers, } \\
\text { et al., } \\
\text { 2001) } \\
\text { Nather } \\
\text { land }\end{array}$ & $\begin{array}{l}\text { Longitudinal study between } \\
1991 \text { and } 1997 \text { with pre- } \\
\text { post observations in which } \\
3993 \text { participants (ages 15- } \\
74 \text { years) were selected } \\
\text { through random sampling } \\
\text { from the population of } \\
27000 \text { those were } \\
\text { Followed up after six years. }\end{array}$ & $\mathrm{N} / \mathrm{R}$ & 85 & $\begin{array}{l}\text { Three items } \\
\text { concerning } \\
\text { Physical } \\
\text { activity } \\
\text { during } \\
\text { leisure time } \\
\text { from valid } \\
\text { measure }\end{array}$ & RLCS & $\begin{array}{l}\text { Logistic } \\
\text { Regressio } \\
\mathrm{n}, \\
\text { Explanat } \\
\text { ory } \\
\text { Analyses, }\end{array}$ & $\begin{array}{l}\text { Individuals with } \\
\text { lower self- } \\
\text { control reported } \\
\text { reduced physical } \\
\text { activity at follow } \\
\text { up measures. }\end{array}$ \\
\hline $\begin{array}{l}\text { (Pfeff } \\
\text { er, et } \\
\text { al., } \\
2017) \\
\text { Grma } \\
\text { ny }\end{array}$ & 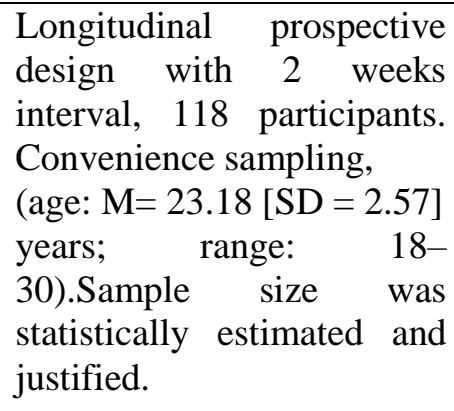 & $\begin{array}{l}46 . \\
6\end{array}$ & 95 & $\begin{array}{l}\text { Single item } \\
\text { vigorous } \\
\text { physical } \\
\text { activity. } \\
\text { Validity was } \\
\text { determined. }\end{array}$ & $\begin{array}{l}\text { Tangney's } \\
\text { SCS }\end{array}$ & $\begin{array}{l}\text { regressio } \\
\mathrm{n} \text { analysis }\end{array}$ & $\begin{array}{l}\text { High trait self- } \\
\text { control predicted } \\
\text { higher physical } \\
\text { activity behavior. }\end{array}$ \\
\hline $\begin{array}{l}\text { Hagg } \\
\text { er, et } \\
\text { al., } \\
\text { 2018) } \\
\text { Finlan } \\
\text { d }\end{array}$ & $\begin{array}{l}\text { Correlational two-wave } \\
\text { Prospective study design, } \\
\text { Total samples size } 3,249 \text {. } \\
\text { Sample one comprised } \\
\text { Finnish males military men } \\
(\mathrm{N}=679) \text { from Finland. } \\
\text { Sample } 2 \text { included } 1072 \\
\text { university students from the } \\
\text { Spain }(\mathrm{N}=282) \text {, UK }(\mathrm{N}= \\
258) \text {, Luxembourg (N = } \\
\text { 172) and Philippines }(\mathrm{N}= \\
\text { 114). } \\
\text { Samples Three and four } \\
\text { consisted of university } \\
\text { students } \\
\text { from the Australia (N = } \\
254) \text { and United Kingdom } \\
\text { (N=150). } \\
\text { Sample } 5 \text { included } 235 \\
\text { students from two public } \\
\text { schools of Finland. } \\
\text { And 280 undergraduate } \\
\text { students from } 5 \text { universities } \\
\text { in Australia were selected } \\
\text { for Sample } 6 \text {. }\end{array}$ & $\begin{array}{l}\text { Bot } \\
\mathrm{h} \\
\text { mal } \\
\mathrm{e} \\
\text { and } \\
\text { fem } \\
\text { ales } \\
\text { wit } \\
\mathrm{h} \\
\text { vari } \\
\text { ed } \\
\text { rati } \\
\mathrm{o} \text { in } \\
\text { sub } \\
\text { sam } \\
\text { ples }\end{array}$ & $\begin{array}{l}3.0 \\
9 \text { to } \\
59 . \\
62\end{array}$ & $\begin{array}{l}\text { Self-reported } \\
\text { participation } \\
\text { in physical } \\
\text { activity, } \\
\text { walking and } \\
\text { physical } \\
\text { activity done } \\
\text { after school } \\
\text { hours }\end{array}$ & $\begin{array}{l}\text { Tangney's } \\
\text { SCS }\end{array}$ & $\begin{array}{l}\text { MANOV } \\
\text { A, chi- } \\
\text { square } \\
\text { tests, } \\
\text { independ } \\
\text { ent t- } \\
\text { tests, } \\
\text { regressio } \\
\text { n } \\
\text { analyses, } \\
\text { the } \\
\text { comparati } \\
\text { ve fit } \\
\text { index } \\
\text { (CFI), the } \\
\text { Tucker- } \\
\text { Lewis } \\
\text { index, } \\
\text { RMSEA }\end{array}$ & $\begin{array}{l}\text { Results from the } \\
\text { samples of } \\
\text { school and } \\
\text { university } \\
\text { students revealed } \\
\text { that there was } \\
\text { positive relation } \\
\text { between physical } \\
\text { activity and trait } \\
\text { self-control. }\end{array}$ \\
\hline
\end{tabular}




\begin{tabular}{|c|c|c|c|c|c|c|c|}
\hline & $\begin{array}{l}\text { Mean ages of the total } \\
\text { sample ranged from } 13 \text { to } \\
21 \text { years. } \\
\text { Follow-up period was } 2,4 \\
\text { and } 8 \text { weeks. }\end{array}$ & & & & & & \\
\hline $\begin{array}{l}\text { (Stork } \\
\text {, et al., } \\
2016) \\
\text { Canad } \\
\text { a }\end{array}$ & $\begin{array}{l}\text { online survey with pre-post } \\
\text { prospective study design } \\
\text { with } 4 \text { weeks period, } \\
\text { sample } 30 \text { university under } \\
\text { graduate students, mean age } \\
\text { of } 18.17 \text { years, sample size } \\
\text { was justified through power } \\
\text { calculations, }\end{array}$ & 23 & 98 & IPAQ-S & $\begin{array}{l}\text { Tangney's } \\
\text { SCS }\end{array}$ & $\begin{array}{l}\text { Multiple } \\
\text { one-tailed } \\
\text { Pearson's } \\
\text { correlatio } \\
\mathrm{n} \\
\text { coefficien } \\
\mathrm{t}, \\
\text { Spearman } \\
\text { 's } \\
\text { correlatio } \\
\mathrm{n} \\
\text { coefficien } \\
\mathrm{t}, \\
\text { regressio } \\
\mathrm{n} \\
\text { analyses }\end{array}$ & $\begin{array}{l}\text { Physical activity } \\
\text { was predicted by } \\
\text { trait self-control } \\
(\mathrm{rs}=0.34, \mathrm{p}= \\
0.04) \text {. } \\
\text { Students with } \\
\text { higher trait self- } \\
\text { control engaged } \\
\text { in exercise for } \\
\text { longer durations } \\
\text { than that of } \\
\text { participants } \\
\text { depicted lower } \\
\text { trait self-control. }\end{array}$ \\
\hline
\end{tabular}

Table 1. C. characteristics of intervention studies examined relation between physical activity and trait selfcontrol. 


\begin{tabular}{|c|c|c|c|c|c|c|c|}
\hline $\begin{array}{l}\text { Autho } \\
\text { r/ } \\
\text { countr } \\
\text { y }\end{array}$ & $\begin{array}{l}\text { Population, sample size and } \\
\text { ages, study design, group } \\
\text { conditions }\end{array}$ & $\begin{array}{l}\text { M } \\
\text { ale } \\
\%\end{array}$ & $\begin{array}{l}\text { Retu } \\
\text { rn } \\
\text { rate } \\
\%\end{array}$ & $\begin{array}{l}\mathrm{PA} \\
\text { measures/PA } \\
\text { interventions }\end{array}$ & $\begin{array}{l}\text { Self- } \\
\text { control } \\
\text { measure } \\
\mathrm{s}\end{array}$ & $\begin{array}{l}\text { Analys } \\
\text { is }\end{array}$ & Findings \\
\hline $\begin{array}{l}\text { (Cecc } \\
\text { hini, } \\
\text { et al,. } \\
\text { 2007) } \\
\text { Spain }\end{array}$ & $\begin{array}{l}\text { Randomized control trial } \\
\text { study design, Population } \\
\text { was } 250,000 \text {. } \\
\text { Total sample size was } 186 \\
\text { middle school students with } \\
\text { ages } 13.6 \text { mean years. } \\
\text { The sample was randomly } \\
\text { divided into three age and } \\
\text { gender matched groups } \\
\text { including experimental } \\
\text { group A, experimental } \\
\text { group B, and a control } \\
\text { group. }\end{array}$ & 49 & 100 & $\begin{array}{l}\text { Playing soccer } \\
\text { for 5 weeks. } \\
\text { Group A } \\
\text { provided with } \\
\text { beginners, } \\
\text { five-a-side } \\
\text { soccer five } \\
\text { level, } \\
\text { Group } \\
\text { provided level } \\
\text { four, and } \\
\text { control group } \\
\text { did not } \\
\text { participated } \\
\text { soccer playing. }\end{array}$ & CASCQ & $\begin{array}{l}\text { Univari } \\
\text { ate } \\
\text { analysi } \\
\mathrm{s}\end{array}$ & $\begin{array}{l}\text { Experimental group } \\
\text { A exhibited } \\
\text { significant } \\
\text { improvements in } \\
\text { delayed } \\
\text { gratification, trait } \\
\text { self-control, and } \\
\text { process self- } \\
\text { regulation as a } \\
\text { result five weeks of } \\
\text { playing soccer than } \\
\text { control group. }\end{array}$ \\
\hline $\begin{array}{l}\text { (Shac } \\
\text { har, et } \\
\text { al., } \\
\text { 2016) } \\
\text { Isreal }\end{array}$ & $\begin{array}{l}\text { Controlled clinical trial } \\
\text { design, } \\
649 \text { Israeli school children } \\
\text { (ages = 9-11 years) equally } \\
\text { divided into sports } \\
\text { intervention group and no } \\
\text { sports intervention control } \\
\text { group. }\end{array}$ & $\begin{array}{l}\text { Ex } \\
\text { per } \\
\text { im } \\
\text { ent } \\
\text { al } \\
\text { gr } \\
\text { ou } \\
\text { p= } \\
82, \\
\text { Co } \\
\text { ntr } \\
\text { ol } \\
\text { gr } \\
\text { ou } \\
\text { p= } \\
70\end{array}$ & 90 & $\begin{array}{l}\text { experimental } \\
\text { group provided } \\
\text { with a } \\
\text { total of } 120 \\
\text { hours of sports } \\
\text { activities after } \\
\text { school hours } \\
\text { for } 24 \text { weeks. } \\
\text { Sports included } \\
\text { soccer, } \\
\text { basketball, } \\
\text { volleyball, and } \\
\text { mini-football } \\
\text { whereas group } \\
\text { Control gro } \\
\text { received no } \\
\text { sport activity. }\end{array}$ & $\begin{array}{l}\text { Tangney } \\
\text { 's SCS }\end{array}$ & $\begin{array}{l}\text { Pearso } \\
\mathrm{n} \\
\text { correlat } \\
\text { ions, } \\
\text { three- } \\
\text { way } \\
\text { multiva } \\
\text { riate } \\
\text { analysi } \\
\text { s of } \\
\text { covaria } \\
\text { nce } \\
\text { (MAN } \\
\text { COVA } \\
\text { ), } \\
\text { linear } \\
\text { regress } \\
\text { ion, } \\
\text { two- } \\
\text { way } \\
\text { MANC } \\
\text { OVA, } \\
\text { and } \\
\text { structur } \\
\text { al }\end{array}$ & $\begin{array}{l}\text { Subjects in the } \\
\text { sports intervention } \\
\text { condition showed } \\
\text { significantly } \\
\text { greater } \\
\text { improvement in } \\
\text { self-control, } \\
\text { significant } \\
\text { reduction } \\
\text { aggression, in } \\
\text { negative emotions, } \\
\text { and hostile } \\
\text { thoughts in } \\
\text { comparison with } \\
\text { participants } \\
\text { control group. in }\end{array}$ \\
\hline
\end{tabular}




\begin{tabular}{|c|c|c|c|c|c|c|c|}
\hline & & & & & & \begin{tabular}{|l} 
equatio \\
$\mathrm{n}$ \\
modeli \\
ng \\
(SEM).
\end{tabular} & \\
\hline $\begin{array}{l}\text { (Cresc } \\
\text { ioni, } \\
\text { et al., } \\
\text { 2011) } \\
\text { USA }\end{array}$ & $\begin{array}{l}12 \text { weak time series single } \\
\text { group design, } \\
86 \text { participants (ages, } \mathrm{M}= \\
26.56, \mathrm{SD}=10.77 \text { ) } \\
\text { participated in weight loss } \\
\text { program for } 12 \text { weeks those } \\
\text { required to record exercise } \\
\text { daily for12 weeks and trait } \\
\text { self-control once in every } \\
\text { two weeks. } \\
\text { The program focused on } \\
\text { reducing weight through } \\
\text { gradually adapting healthy } \\
\text { food and increased physical } \\
\text { activity. Exercises included } \\
\text { walking, jogging, aerobic } \\
\text { dance, cycling, and } \\
\text { swimming. }\end{array}$ & $\begin{array}{l}29 . \\
1\end{array}$ & \begin{tabular}{|l}
62.3 \\
1
\end{tabular} & $\begin{array}{l}\text { BMI, } \\
\text { Five questions } \\
\text { regarding } \\
\text { physical } \\
\text { activity drawn } \\
\text { from previous } \\
\text { research }\end{array}$ & $\begin{array}{l}\text { Tangney } \\
\text { 's SCS }\end{array}$ & \begin{tabular}{|l} 
Regres \\
sion \\
analysi \\
s, \\
Multile \\
vel \\
modeli \\
ng
\end{tabular} & $\begin{array}{l}\text { At baseline, } \\
\text { participants } \\
\text { exhibited higher } \\
\text { trait self-control } \\
\text { appeared to engage } \\
\text { in more physical } \\
\text { activity compared } \\
\text { with subjects } \\
\text { yielded lower trait } \\
\text { self-control. } \\
\text { Subjects } \\
\text { demonstrated } \\
\text { greater trait self- } \\
\text { control } \\
\text { significantly } \\
\text { increased amount } \\
\text { of exercise more } \\
\text { during } 12 \text { weeks of } \\
\text { intervention period } \\
\text { as compared with } \\
\text { subjects exhibited } \\
\text { lower trait self- } \\
\text { control. }\end{array}$ \\
\hline \multicolumn{8}{|c|}{$\begin{array}{l}\text { Abbreviations: PA = physical activity; BMI= body mass index; LTP = leisure time physical activity; BREQ-2 } \\
=\text { The Behavioral Regulation Exercise Questionnaire-2; SRHI = Self-Report Habit Index; SQUASH =Short- } \\
\text { Questionnaire to Assess Health-enhancing physical activity; PAQ = physical activity questionnaire; IPAQ-S = } \\
\text { International Physical Activity Questionnaire, Short Form; SC = self-control; SCS = Tangney Self-Control Scale; } \\
\text { SRQ = Self-Regulation Questionnaire; NEO-C = self-discipline; Rosenbaum,s SCS = Rosenbaum,s Self-Control } \\
\text { Scale; SCRS = Self-Control Rating Scale; RLCS = Rotter's locus of Control Scale; CASCQ = The child and } \\
\text { adolescent self-control questionnaire; }\end{array}$} \\
\hline
\end{tabular}

\subsection{Quality of the Studies}

\subsubsection{Cross-Sectional Studies $(\mathrm{N}=13)$}

In this review, 13 cross-sectional studies were identified that were classified as good $(\mathrm{N}=8)$ or satisfactory $(\mathrm{N}=5)$ quality rating (See table 2. A. for details). All of the cross-sectional studies included in this review selected the participants using convenience sampling approach. Among 13 selected studies, seven studies were classified as good quality rating. These studies used self-reported valid and reliable physical activity and trait self-control measures, controlled for at least most important confounding factors, reported sufficient response rate except two(Beenackers, Oude Groeniger, van Lenthe, \& Kamphuis, 2017; Kinnunen, Suihko, Hankonen, Absetz, \& Jallinoja, 2012) studies, sufficiently representative sample, satisfactory sample size with the exception of three(Chen et al., 2018; Gillebaart \& Adriaanse, 2017; Toering \& Jordet, 2015) studies, and applied appropriate statistical tests for analysis. Five of the selected cross-sectional studies were rated as satisfactory level of study quality(Allom et al., 2016; Briki, 2016; Junger \& van Kampen, 2010; Park et al., 2016; Shachaf \& Katz, 2014; Wills, Isasi, Mendoza, \& Ainette, 2007). These studies had insufficient representativeness of samples and 
unsatisfactory sample sizes. However, these studies did use valid and reliable physical activity and trait self-control measures, controlled the most important confounding factors, had sufficient level of participant's response rate and used appropriate statistical tests for analysis.

Table 2. A. Quality assessment of the cross-sectional included studies based on The Newcastle-Ottawa Scale (NOS) adapted for cross-sectional studies.

\begin{tabular}{|c|c|c|c|c|c|c|c|c|c|}
\hline \multirow[t]{3}{*}{ Study } & \multicolumn{4}{|l|}{ Selection } & \multirow{2}{*}{$\begin{array}{l}\text { Comparabi } \\
\text { lity } \\
5\end{array}$} & \multicolumn{2}{|l|}{ Outcome } & & \\
\hline & 1 & 2 & 3 & 4 & & 6 & 7 & & \\
\hline & $\begin{array}{l}\text { Represen } \\
\text { tativenes } \\
\mathrm{s} \text { of the } \\
\text { sample } \\
*\end{array}$ & $\begin{array}{l}\text { Sam } \\
\text { ple } \\
\text { size } \\
*\end{array}$ & $\begin{array}{l}\text { Non- } \\
\text { respon } \\
\text { dents } \\
*\end{array}$ & $\begin{array}{l}\text { Ascertainme } \\
\mathrm{nt} \text { of the } \\
\text { exposure } \\
* *\end{array}$ & $\begin{array}{l}\text { Confoundi } \\
\text { ng factors } \\
\text { are } \\
\text { controlled } \\
* *\end{array}$ & $\begin{array}{l}\text { Assessm } \\
\text { ent of the } \\
\text { outcome } \\
* *\end{array}$ & $\begin{array}{l}\text { Statist } \\
\text { ical } \\
\text { test } \\
*\end{array}$ & $\begin{array}{l}\text { Qualit } \\
y \\
\text { score } \\
\text { out of } \\
10\end{array}$ & $\begin{array}{l}\text { Qualit } \\
\mathrm{y} \\
\text { rating }\end{array}$ \\
\hline $\begin{array}{l}\text { Wills, et al,. } \\
2007\end{array}$ & - & - & $*$ & $* *$ & $*$ & - & $*$ & 5 & $\begin{array}{l}\text { Satisf } \\
\text { actory }\end{array}$ \\
\hline $\begin{array}{l}\text { Junger, et al., } \\
2010\end{array}$ & - & - & $*$ & $* *$ & $* *$ & - & $*$ & 6 & $\begin{array}{l}\text { Satisf } \\
\text { actory }\end{array}$ \\
\hline $\begin{array}{l}\text { Kinnunen, et } \\
\text { al,. (2012) }\end{array}$ & $*$ & $*$ & - & $* *$ & $*$ & $*$ & $*$ & 7 & Good \\
\hline $\begin{array}{l}\text { Shachaf, et al., } \\
2014\end{array}$ & - & - & $*$ & $* *$ & - & $*$ & $*$ & 5 & $\begin{array}{l}\text { Satisf } \\
\text { actory }\end{array}$ \\
\hline $\begin{array}{l}\text { Toering, et al., } \\
2015\end{array}$ & $*$ & - & $*$ & $* *$ & $* *$ & $*$ & $*$ & 8 & Good \\
\hline $\begin{array}{l}\text { Park, et al., } \\
2016\end{array}$ & - & - & $*$ & $* *$ & - & $*$ & $*$ & 5 & $\begin{array}{l}\text { Satisf } \\
\text { actory }\end{array}$ \\
\hline $\begin{array}{l}\text { Tedesqui, et al., } \\
\text { (2017) }\end{array}$ & $*$ & $*$ & $*$ & $* *$ & $*$ & $*$ & $*$ & 8 & Good \\
\hline $\begin{array}{l}\text { Chen, et al., } \\
(2018)\end{array}$ & $*$ & - & $*$ & $* *$ & $* *$ & $*$ & $*$ & 8 & Good \\
\hline $\begin{array}{l}\text { Chen, et al., } \\
2019\end{array}$ & $*$ & $*$ & $*$ & $* *$ & $*$ & $*$ & $*$ & 8 & Good \\
\hline $\begin{array}{l}\text { Briki, } \quad \text { w., } \\
(2016)\end{array}$ & - & - & $*$ & $* *$ & $* *$ & - & $*$ & 6 & $\begin{array}{l}\text { Satisf } \\
\text { actory }\end{array}$ \\
\hline $\begin{array}{l}\text { Gillebaart , at } \\
\text { al., } 2017\end{array}$ & $*$ & - & $*$ & $* *$ & $*$ & $*$ & $*$ & 7 & Good \\
\hline $\begin{array}{l}\text { Beenackers, et } \\
\text { al., } \\
2017\end{array}$ & $*$ & $*$ & - & $* *$ & $* *$ & $*$ & $*$ & 8 & Good \\
\hline $\begin{array}{l}\text { Allom, et al., } \\
2016\end{array}$ & - & - & $*$ & $* *$ & $*$ & $*$ & $*$ & 6 & $\begin{array}{l}\text { Satisf } \\
\text { actory }\end{array}$ \\
\hline $\begin{array}{l}\text { Quality Rating pr } \\
\text { Very Good Studi } \\
\text { Good Studies: } 7 \\
\text { Satisfactory Stud } \\
\text { Unsatisfactory St }\end{array}$ & $\begin{array}{l}\text { ocedure: } \\
\text { es: } 9-10 \text { poi } \\
\text { points } \\
\text { es: } 5-6 \text { poil } \\
\text { udies: } 0 \text { to }\end{array}$ & oint & & & & & & & \\
\hline
\end{tabular}

\subsubsection{Longitudinal $(\mathbf{N}=5)$ and Intervention Studies $(\mathbf{N}=3$}

Out of five admissible longitudinal studies, one study is categorized in strong rating having low risk of bias with no weak rating in any of the component of quality assessment tool (Stork, Graham, Bray, \& Martin Ginis, 2017).The details regarding quality ratings are depicted in Table. 2. B and $\mathrm{C}$ respectively.In addition, four out of five longitudinal studies were classified in moderate quality ratings (Droomers, Schrijvers, \& Mackenbach, 2001; Martin S Hagger et al., 2018; Pfeffer \& Strobach, 2017;Schöndube, Bertrams, Sudeck, \& Fuchs, 2017). These 
studies were characterized by following limitations: weak rating in selection bias $2 / 5$ (Pfeffer \& Strobach, 2017; Schöndube et al., 2017) and lower response rate (2/5) (Droomers et al., 2001; Martin S Hagger et al., 2018).

With regard to quality of the intervention studies, we identified three studies with over all strong quality rating (Cecchini et al., 2007; Shachar et al., 2016; Will Crescioni et al., 2011). These studies adequately minimized the selection bias in study samples (2/3) (Cecchini et al., 2007; Shachar et al., 2016), used strong study designs(2/3 (Cecchini et al., 2007; Will Crescioni et al., 2011), controlled for the major confounding factors (2/3)(Shachar et al., 2016; Will Crescioni et al., 2011), used valid and reliable physical activity and trait self-control measures (3/3)(Cecchini et al., 2007; Shachar et al., 2016; Will Crescioni et al., 2011), and had low withdrawal rates (2/3) (Cecchini et al., 2007; Shachar et al., 2016). None of the intervention studies admissible to this article had weak rating in any of the component of quality assessment criteria.

Table 2.B. Quality assessment of longitudinal studies based on Effective Public Health Practice Project (EPHPP) Quality Assessment Tool

\begin{tabular}{|c|c|c|c|c|c|c|c|c|c|}
\hline Study & $\begin{array}{l}\text { Selectio } \\
\mathrm{n} \text { bias }\end{array}$ & $\begin{array}{l}\text { B } \\
\text { Study } \\
\text { Desig } \\
n\end{array}$ & $\begin{array}{l}\text { C } \\
\text { Confounder } \\
\mathrm{S}\end{array}$ & $\begin{array}{l}\mathrm{D} \\
\text { Blindin } \\
\mathrm{g}\end{array}$ & $\begin{array}{l}\mathrm{E} \\
\text { Data } \\
\text { collectio } \\
\mathrm{n} \\
\text { methods }\end{array}$ & $\begin{array}{l}\text { F } \\
\text { Withdraw } \\
\text { al }\end{array}$ & $\begin{array}{l}\mathrm{G} \\
\text { Interventio } \\
\mathrm{n} \text { integrity }\end{array}$ & $\begin{array}{l}\mathrm{H} \\
\text { Analysi } \\
\mathrm{S}\end{array}$ & $\begin{array}{l}\text { Overall } \\
\text { rating }\end{array}$ \\
\hline $\begin{array}{l}\text { Schondub } \\
\text { e, et al., } \\
(2017)\end{array}$ & $\mathrm{w}$ & $\mathrm{m}$ & $\mathrm{m}$ & $\mathrm{m}$ & $\mathrm{S}$ & $\mathrm{s}$ & $\mathrm{n} / \mathrm{a}$ & $\mathrm{y}$ & $\begin{array}{l}\text { moderat } \\
\mathrm{e}\end{array}$ \\
\hline $\begin{array}{l}\text { Droomers, } \\
\text { et al., } \\
2001\end{array}$ & $\mathrm{~s}$ & $\mathrm{~s}$ & $\mathrm{~s}$ & $\mathrm{~m}$ & $\mathrm{~s}$ & $\mathrm{w}$ & $\mathrm{n} / \mathrm{a}$ & $\mathrm{y}$ & $\begin{array}{l}\text { moderat } \\
\mathrm{e}\end{array}$ \\
\hline $\begin{array}{l}\text { Pfeffer, et } \\
\text { al., } 2017\end{array}$ & $\mathrm{w}$ & $\mathrm{m}$ & $\mathrm{m}$ & $\mathrm{m}$ & $\mathrm{s}$ & $\mathrm{s}$ & $\mathrm{n} / \mathrm{a}$ & $y$ & $\begin{array}{l}\text { moderat } \\
\mathrm{e}\end{array}$ \\
\hline $\begin{array}{l}\text { Stork, et } \\
\text { al., 2016 }\end{array}$ & $\mathrm{m}$ & $\mathrm{m}$ & $\mathrm{m}$ & $\mathrm{m}$ & $\mathrm{s}$ & $\mathrm{s}$ & $\mathrm{n} / \mathrm{a}$ & $y$ & strong \\
\hline $\begin{array}{l}\text { Hagger, et } \\
\text { al., } 2018\end{array}$ & $\mathrm{~m}$ & $\mathrm{~s}$ & $\mathrm{~m}$ & $\mathrm{~m}$ & $\mathrm{~s}$ & $\mathrm{w}$ & $\mathrm{n} / \mathrm{a}$ & $y$ & $\begin{array}{l}\text { moderat } \\
\mathrm{e}\end{array}$ \\
\hline \multicolumn{10}{|c|}{ Table. 2. C. Quality assessment of intervention studies } \\
\hline $\begin{array}{l}\text { Shachar, } \\
\text { et al., } \\
(2016)\end{array}$ & $\mathrm{s}$ & $\mathrm{m}$ & $\mathrm{s}$ & $\mathrm{m}$ & $\mathrm{m}$ & $\mathrm{s}$ & $\mathrm{y}$ & $\mathrm{y}$ & strong \\
\hline $\begin{array}{l}\text { Cecchini, } \\
\text { et al,. } \\
2007\end{array}$ & $\mathrm{~s}$ & $\mathrm{~s}$ & $\mathrm{~m}$ & $\mathrm{~m}$ & $\mathrm{~s}$ & $\mathrm{~s}$ & $\mathrm{y}$ & $\mathrm{y}$ & Strong \\
\hline \begin{tabular}{l}
\multicolumn{2}{l}{ Crescioni, } \\
et al., \\
2011
\end{tabular} & $\mathrm{~m}$ & $\mathrm{~s}$ & $\mathrm{~s}$ & $\mathrm{~m}$ & $\mathrm{~s}$ & $\mathrm{~m}$ & $y$ & $\mathrm{y}$ & strong \\
\hline $\begin{array}{l}\text { Rating scale } \\
\text { Criteria for } 0 \\
\text { Strong= No } \\
\text { Moderate = } \\
\text { Weak = Two }\end{array}$ & $\begin{array}{l}\text { Strong = } \\
\text { verall rati } \\
\text { weak ratin } \\
\text { One weak } \\
\text { or more }\end{array}$ & $\begin{array}{l}\text { S, Mode } \\
\text { ig of the } \\
\text { ating } \\
\text { yeak ratil }\end{array}$ & $\begin{array}{l}\text { ate }=\mathrm{M} \text {, Weal } \\
\text { study }\end{array}$ & $=\mathrm{W}$ & & & & & \\
\hline
\end{tabular}

\section{Discussions}

Lack of self-control constitute the likelihood of increased aggression, violent behavior, crimes and delinquencies, sedentary behavior, addictive tendencies, and inactivity that may increase wide range of social and health related 
problems. In this connection, interventional strategies are crucially needed that may improve self-control in individuals with low level of self-control. Therefore, this study sought to systematically analyze the efficacy of physical activity, sports participation, and exercise to enhance self-control potentials. As per our knowledge, this is the first article that synthesized the findings from studies with sufficient quality and methodological rigor that tried to present findings on the said area of research. Thirteen cross-sectional studies with good (7/13) or satisfactory (6/13) quality ratings, five longitudinal studies with strong (1/5) or moderate (4/5) quality ratings, and three intervention studies with strong overall quality ratings presented findings in support of significantly positive relationship of physical activity, exercise, and sports participation with trait self-control and the vice versa.The consistency in findings from cross-sectional, longitudinal, and intervention studies provide sufficient evidence in favor of positive relation between physical activity and trait self-control. No study found inverse relation among these variables. Based on these findings, it can be suggested that participation in exercise and sports activities may be considered as a therapeutic mean to enhance self-control in individuals with low trait self-control and vice versa.

These findings are congruent with previous research indicating that participation in sports and physical activities foster positive psychological traits(Shachaf \& Katz, 2014), and emotional well-being (Donaldson \& Ronan, 2006). Some studies included in this review proposed the mechanismsthat might account for the positive relationship between physical activity and trait self-control. For example, findings from an intervention study conducted by Shachar et al. (2016), proposed that improvement in positive and negative emotions, and reduction in physical aggression as a result of participation in sport activities likely to foster self-control. Other studies suggested that participation in physical activities likely to increase self- efficacy, decrease aggression (Chen et al., 2019), and constitute positive habits (Gillebaart \& Adriaanse, 2017) that in return might contribute improving trait self-control.

\section{Strengths and Limitations of this Study}

This study performed systematic literature search in the major research databases and presented well-structured and extensive synthesis of evidence. We evaluated the studies using valid, reliable and extensively used study quality assessment tools that were more appropriate for cross-sectional, longitudinal and intervention studies. Three intervention studies and one longitudinal study with strong methodologies and lower risk of biases added strength to the findings of this study. However, this study had some following limitations. For example, some of the included cross-sectional and longitudinal studies had moderate level of methodological quality that had risk of biased findings.

\subsection{Future Research}

Randomized control trial research design is considered as the gold standard design with respect to methodological quality. Experimental studies with randomized controlled trial (RCT) are limited on this topic as we could identify only one high quality study that used RCT design. Further research should focus on high quality studies with these research designs. Cross-sectional studies with larger sample sizes using systematic sampling techniques are also needed. Previous research heavily relied on using self-reported physical activity measures. Future research should use objective measures of physical activity while assessing relationship of physical activity and the ability of selfcontrol. The link between type and form of physical activity and trait self-control can be another important venue of research that should be taken into consideration in the future.

\section{Conclusion and Practical Implications}

Individuals with criminal record have shown low level of trait self-control, hence, Policy makers should consider planning of exercise programs in jails for prisoners to engage them in sporting and exercise programs to enhance self-control levels in these individuals that could further reduce the tendency of criminology. Moreover, in current years, involvement in violent, bullying, aggressive and wide range of addictive behaviors has increased significantly among youth in education institutions. This posed a serious threat to peaceful learning environment of these institutions. Youth with these negative tendencies can be treated by offering special intervention programs including physical activities. This may help to improve self-control that further tends to reduce these negative behaviors among youth. On the other hand, dealing with the problem of inactivity or low level of physical activity has become major concern across the world. Since, higher level of trait self-control is related with increased physical activity, therapeutic intervention that could foster self-control can be helpful to increase level of physical activity in peoples with sedentary behavior. In sum, physical activity can be suggested as a potential medium of improving trait self-control. These recommendations have important implication for the professionals related with 
criminology, health, psychology, and education. Policy makers can consider these guidelines while making decisions in these areas.

\section{References}

Allom, V., Panetta, G., Mullan, B., \& Hagger, M. S. (2016). Self-report and behavioural approaches to the measurement of self-control: Are we assessing the same construct? Personality and Individual Differences, 90, 137-142.

Armijo-Olivo, S., Stiles, C. R., Hagen, N. A., Biondo, P. D., \& Cummings, G. G. (2012). Assessment of study quality for systematic reviews: a comparison of the Cochrane Collaboration Risk of Bias Tool and the Effective Public Health Practice Project Quality Assessment Tool: methodological research. Journal of Evaluation in Clinical Practice, 18(1), 12-18. doi: 10.1111/j.1365-2753.2010.01516.x

Baer, M. M., LaCroix, J. M., Browne, J. C., Hassen, H. O., Perera, K. U., Soumoff, A., . . . GhahramanlouHolloway, M. (2018). Impulse control difficulties while distressed: A facet of emotion dysregulation links to Non-Suicidal Self-Injury among psychiatric inpatients at military treatment facilities. Psychiatry research, 269, 419-424. doi: https://doi.org/10.1016/j.psychres.2018.08.082

Beenackers, M. A., Oude Groeniger, J., van Lenthe, F. J., \& Kamphuis, C. B. (2017). The role of financial strain and self-control in explaining health behaviours: the GLOBE study. The European Journal of Public Health, 28(4), 597-603.

Bobbio, A., Arbach, K., \& Vazsonyi, A. T. (2019). Self-Control and Deviance: A Test of the General Theory of Crime in Argentina. Victims \& Offenders, 14(1), 119-142. doi: 10.1080/15564886.2018.1552222

Briki, W. (2016). Motivation toward physical exercise and subjective wellbeing: the mediating role of trait selfcontrol. Frontiers in psychology, 7, 1546.

Cecchini, J. A., Montero, J., Alonso, A., Izquierdo, M., \& Contreras, O. (2007). Effects of personal and social responsibility on fair play in sports and self-control in school-aged youths. European Journal of sport science, 7(4), 203-211.

Chen, X., Li, B., Xie, J., Li, Y., \& Zhang, G. (2018). The Big Five, Self-efficacy, and Self-control in Boxers. BioRxiv, 361295.

Chen, X., Zhang, G., Yin, X., Li, Y., Cao, G., Gutiérrez-García, C., \& Guo, L. (2019). The Relationship Between Self-Efficacy and Aggressive Behavior in Boxers: The Mediating Role of Self-Control. Frontiers in psychology, 10.

Crick, N. R., \& Dodge, K. A. (1994). A review and reformulation of social information-processing mechanisms in children's social adjustment. Psychological bulletin, 115(1), 74.

De Ridder, D., \& Gillebaart, M. (2017). Lessons learned from trait self-control in well-being: Making the case for routines and initiation as important components of trait self-control. Health psychology review, 11(1), 8999.

De Ridder, D. T., \& Lensvelt-Mulders, G. (2018). Taking stock of self-control: A meta-analysis of how trait selfcontrol relates to a wide range of behaviors Self-Regulation and Self-Control (pp. 221-274): Routledge.

Donaldson, S. J., \& Ronan, K. R. (2006). THE EFFECTS OF SPORTS PARTICIPATION ON YOUNG ADOLESCENTS'EMOTIONAL WELL-BEING. Adolescence, 41(162).

Droomers, M., Schrijvers, C. T. M., \& Mackenbach, J. (2001). Educational level and decreases in leisure time physical activity: predictors from the longitudinal GLOBE study. Journal of Epidemiology \& Community Health, 55(8), 562-568.

Duckworth, A. L., Gendler, T. S., \& Gross, J. J. (2016). Situational Strategies for Self-Control. Perspectives on psychological science : a journal of the Association for Psychological Science, 11(1), 35-55. doi: $10.1177 / 1745691615623247$

Elias, M. J., Zins, J. E., \& Weissberg, R. P. (1997). Promoting social and emotional learning: Guidelines for educators: Ascd.

Fan, M., \& Jin, Y. (2013). Obesity and Self-control: Food Consumption, Physical Activity, and Weight-loss Intention. Applied Economic Perspectives and Policy, 36(1), 125-145. doi: 10.1093/aepp/ppt034

Gillebaart, M. (2018). The 'Operational' Definition of Self-Control. [Perspective]. Frontiers in psychology, 9(1231). doi: 10.3389/fpsyg.2018.01231

Gillebaart, M., \& Adriaanse, M. A. (2017). Self-control predicts exercise behavior by force of habit, a conceptual replication of Adriaanse et al.(2014). Frontiers in psychology, 8, 190.

Hagger, M. S., Hankonen, N., Kangro, E.-M., Lintunen, T., Pagaduan, J., Polet, J., . . . Hamilton, K. Trait SelfControl, Social Cognition Constructs, and Intentions: Correlational Evidence for Mediation and Moderation Effects in Diverse Health Behaviours. Applied Psychology: Health and Well-Being, 0(0). doi: 
10.1111/aphw.12153

Hagger, M. S., Hankonen, N., Kangro, E. M., Lintunen, T., Pagaduan, J., Polet, J., . . Hamilton, K. (2018). Trait Self-Control, Social Cognition Constructs, and Intentions: Correlational Evidence for Mediation and Moderation Effects in Diverse Health Behaviours. Applied Psychology: Health and Well-Being.

Hallquist, M. N., Hipwell, A. E., \& Stepp, S. D. (2015). Poor self-control and harsh punishment in childhood prospectively predict borderline personality symptoms in adolescent girls. Journal of abnormal psychology, 124(3), 549-564. doi: 10.1037/abn0000058

Herzog, R., Álvarez-Pasquin, M. J., Díaz, C., Del Barrio, J. L., Estrada, J. M., \& Gil, Á. (2013). Are healthcare workers' intentions to vaccinate related to their knowledge, beliefs and attitudes? A systematic review. BMC public health, 13(1), 154.

Isen, A. M., \& Reeve, J. (2005). The influence of positive affect on intrinsic and extrinsic motivation: Facilitating enjoyment of play, responsible work behavior, and self-control. Motivation and emotion, 29(4), 295-323.

Junger, M., \& van Kampen, M. (2010). Cognitive ability and self-control in relation to dietary habits, physical activity and bodyweight in adolescents. International journal of behavioral nutrition and physical activity, $7(1), 22$.

Kim, E. J., Namkoong, K., Ku, T., \& Kim, S. J. (2008). The relationship between online game addiction and aggression, self-control and narcissistic personality traits. European Psychiatry, 23(3), 212-218.

Kinnunen, M. I., Suihko, J., Hankonen, N., Absetz, P., \& Jallinoja, P. (2012). Self-control is associated with physical activity and fitness among young males. Behavioral Medicine, 38(3), 83-89.

Lubans, D. R., Plotnikoff, R. C., \& Lubans, N. J. (2012). Review: A systematic review of the impact of physical activity programmes on social and emotional well-being in at-risk youth. Child and Adolescent Mental Health, 17(1), 2-13. doi: 10.1111/j.1475-3588.2011.00623.x

Mackenbach, J. D., Beenackers, M. A., Noordzij, J. M., Oude Groeniger, J., Lakerveld, J., \& van Lenthe, F. J. (2019). The Moderating Role of Self-Control and Financial Strain in the Relation between Exposure to the Food Environment and Obesity: The GLOBE Study. International Journal of Environmental Research and Public Health, 16(4), 674.

Malouf, E. T., Schaefer, K. E., Witt, E. A., Moore, K. E., Stuewig, J., \& Tangney, J. P. (2014). The brief selfcontrol scale predicts jail inmates' recidivism, substance dependence, and post-release adjustment. [Research Support, N I H , Extramural]. Pers Soc Psychol Bull, 40(3), 334-347.

Mann, T., De Ridder, D., \& Fujita, K. (2013). Self-regulation of health behavior: social psychological approaches to goal setting and goal striving. Health Psychology, 32(5), 487.

Nęcka, E., Korona-Golec, K., Hlawacz, T., Nowak, M., \& Gruszka-Gosiewska, A. (2018). The relationship between self-control and temperament: a contribution to the self-control definition debate. Current Issues in Personality Psychology, 7(1), 24-31.

Nieman, P. (2002). Psychosocial aspects of physical activity. Paediatrics \& Child Health, 7(5), 309-312. doi: $10.1093 / \mathrm{pch} / 7.5 .309$

Oaten, M., \& Cheng, K. (2006). Longitudinal gains in self-regulation from regular physical exercise. British Journal of Health Psychology, 11(4), 717-733. doi: 10.1348/135910706x96481

Özdemir, Y., Kuzucu, Y., \& Ak, Ş. (2014). Depression, loneliness and Internet addiction: How important is low self-control? Computers in Human Behavior, 34, 284-290.

Park, J.-A., Park, M.-H., Shin, J.-H., Li, B., Rolfe, D. T., Yoo, J.-Y., \& Dittmore, S. W. (2016). Effect of sports participation on internet addiction mediated by self-control: A case of korean adolescents. Kasetsart Journal of Social Sciences, 37(3), 164-169.

Penedo, F. J., \& Dahn, J. R. (2005). Exercise and well-being: a review of mental and physical health benefits associated with physical activity. Current opinion in psychiatry, 18(2), 189-193.

Pfeffer, I., \& Strobach, T. (2017). Executive functions, trait self-control, and the intention-behavior gap in physical activity behavior. Journal of sport \& exercise psychology, 39(4), 277-292.

Pilarska, A., \& Baumeister, R. F. (2018). Psychometric properties and correlates of the Polish version of the SelfControl Scale (SCS). Polish Psychological Bulletin.

Piquero, A. R., Jennings, W. G., Farrington, D. P., Diamond, B., \& Gonzalez, J. M. R. (2016). A meta-analysis update on the effectiveness of early self-control improvement programs to improve self-control and reduce delinquency. [journal article]. Journal of Experimental Criminology, 12(2), 249-264. doi: 10.1007/s11292$016-9257-\mathrm{z}$

Project, E. P. H. P. (1998). Quality Assessment Tool for Quantitative Studies. EPHPP, from https://merst.ca/wpcontent/uploads/2018/02/quality-assessment-tool_2010.pdf

Rowland, Z., Wenzel, M., \& Kubiak, T. (2016). The effects of computer-based mindfulness training on Self-control 
and Mindfulness within Ambulatorily assessed network Systems across Health-related domains in a healthy student population (SMASH): study protocol for a randomized controlled trial. Trials, 17(1), 570-570. doi: 10.1186/s13063-016-1707-4

Scarpa, A., \& Reyes, N. M. (2011). Improving emotion regulation with CBT in young children with high functioning autism spectrum disorders: A pilot study. Behavioural and cognitive psychotherapy, 39(4), 495-500.

Schöndube, A., Bertrams, A., Sudeck, G., \& Fuchs, R. (2017). Self-control strength and physical exercise: an ecological momentary assessment study. Psychology of Sport and Exercise, 29, 19-26.

Scully, D., Kremer, J., Meade, M. M., Graham, R., \& Dudgeon, K. (1998). Physical exercise and psychological well being: a critical review. British journal of sports medicine, 32(2), 111-120.

Seibert, G. S., May, R. W., Fitzgerald, M. C., \& Fincham, F. D. (2016). Understanding school burnout: Does selfcontrol matter? Learning and Individual Differences, 49, 120-127.

Shachaf, M., \& Katz, Y. J. (2014). The relationship between high school students' participation in sporting activity and personality variables. Athens Journal of Sports, 1(1), 35-43.

Shachar, K., Ronen-Rosenbaum, T., Rosenbaum, M., Orkibi, H., \& Hamama, L. (2016). Reducing child aggression through sports intervention: The role of self-control skills and emotions. Children and Youth Services Review, 71, 241-249.

Stein, E., Thurston, W. P., \& Mather, J. N. (1997). Three-dimensional geometry and topology (Vol. 1): Princeton university press.

Stork, M. J., Graham, J. D., Bray, S. R., \& Martin Ginis, K. A. (2017). Using self-reported and objective measures of self-control to predict exercise and academic behaviors among first-year university students. Journal of Health Psychology, 22(8), 1056-1066.

Tangney, J. P., Baumeister, R. F., \& Boone, A. L. (2004). High self-control predicts good adjustment, less pathology, better grades, and interpersonal success. Journal of personality, 72(2), 271-324.

Tao, F. B., Xu, M. L., Kim, S. D., Sun, Y., Su, P. Y., \& Huang, K. (2007). Physical activity might not be the protective factor for health risk behaviours and psychopathological symptoms in adolescents. Journal of Paediatrics and Child Health, 43(11), 762-767. doi: 10.1111/j.1440-1754.2007.01217.x

Tedesqui, R. A., \& Young, B. W. (2017). Associations between self-control, practice, and skill level in sport expertise development. Research Quarterly for Exercise and Sport, 88(1), 108-113.

Thomas, B., Ciliska, D., Dobbins, M., \& Micucci, S. (2004). A process for systematically reviewing the literature: providing the research evidence for public health nursing interventions. Worldviews on Evidence-Based Nursing, 1(3), 176-184.

Toering, T., \& Jordet, G. (2015). Self-control in professional soccer players. Journal of applied sport psychology, 27(3), 335-350.

Verplanken, B., \& Orbell, S. (2003). Reflections on past behavior: a self-report index of habit strength 1. Journal of applied social psychology, 33(6), 1313-1330.

von Hammerstein, C., Miranda, R., Aubin, H.-J., Romo, L., Khazaal, Y., Benyamina, A., \& Luquiens, A. (2018). Mindfulness and Cognitive Training in a CBT-resistant Patient With Gambling Disorder: A Combined Therapy to Enhance Self-control. Journal of addiction medicine, 12(6), 484-489.

Will Crescioni, A., Ehrlinger, J., Alquist, J. L., Conlon, K. E., Baumeister, R. F., Schatschneider, C., \& Dutton, G. R. (2011). High trait self-control predicts positive health behaviors and success in weight loss. Journal of Health Psychology, 16(5), 750-759.

Wills, T. A., Isasi, C. R., Mendoza, D., \& Ainette, M. G. (2007). Self-control constructs related to measures of dietary intake and physical activity in adolescents. Journal of Adolescent Health, 41(6), 551-558. 
